\title{
Riemannian Manifolds and Gauge Theory
}

\author{
Hyun Seok Yang ${ }^{* \dagger}$ \\ Institute for the Early Universe, Ewha Womans University, Seoul 120-750, Korea \\ Center for Quantum Spacetime, Sogang University, Seoul 121-741, Korea \\ E-mail: hsyangdsogang.ac.kr
}

Gravity and gauge theory share several analogous structures besides some critical differences. A decisive common structure, on one hand, is that Einstein gravity can be formulated as a gauge theory of Lorentz group where spin connections play a role of gauge fields and Riemann curvature tensors correspond to their field strengths. On the other hand, a crucial difference is that the action of Einstein gravity is linear in curvature tensors in contrast to the quadratic action of YangMills gauge theory. Through a systematic gauge theory formulation of gravity, we will clarify a topological origin to shed light on why the action of gravity is linear in curvature tensors contrary to gauge theory. In particular, we will identify the gauge theory object corresponding to Einstein manifolds in four dimensions and to Calabi-Yau manifolds in six dimensions, which directly reveals the topological aspects of Riemannian manifolds.

Proceedings of the Corfu Summer Institute 2011 School and Workshops on Elementary Particle Physics and Gravity

September 4-18, 2011

Corfu, Greece

\footnotetext{
* Speaker.

$\dagger$ This research was supported by Basic Science Research Program through the National Research Foundation of Korea (NRF) funded by the Ministry of Education, Science and Technology (2011-0010597) and by the RP-Grant 2010 of Ewha Womans University.
} 


\section{Introduction}

The physics on a curved spacetime becomes more transparent when expressed in a locally inertial frame and it is even indispensable when one want to couple spinors to gravity since spinors in $d$-dimensions form a representation of $S O(d)$ rather than $G L(d, \mathbb{R})$. On the orthonormal frame bundle, a Riemannian metric on spacetime manifold $M$ is replaced by a local basis for the tangent bundle $T M$, which is orthonormal tangent vectors $E_{A}(A=1, \cdots, d)$ on $M$. But, in any vector space, there is a freedom for the choice of basis and physical observables are independent of the arbitrary choice of an orthonormal frame. As in any other gauge theory with local gauge invariance, to achieve local Lorentz invariance requires introducing a gauge field $\omega^{A}{ }_{B}$ of the Lorentz group $S O(d)$. The gauge field of the local Lorentz group is called the spin connection [四]. In the end, $d$-dimensional Einstein gravity can be formulated as a gauge theory of $S O(d)$ Lorentz group where spin connections play a role of gauge fields and Riemann curvature tensors correspond to their field strengths.

In spite of the close analogy between gravity and gauge theory, there also exist acute differences between them. It is worthwhile to notice that the fundamental variable in gravity is the orthonormal tangent vectors $E_{A}$, the so-called (inverse) vielbeins, rather than spin connections. The spin connections are determined by the vielbeins via the torsion free condition [四]. In other words, the spin connections have their own gauge fields, i.e. vielbeins, though they are $S O(d)$ gauge fields from the gauge theory point of view. As a result, the Riemann curvature tensor already contains second-order derivatives of the Riemannian metric. Therefore the action of Einstein gravity must be linear in curvature tensors for the theory to be unitary because higher order terms such as a quadratic term in curvature tensors will generate ghost fields in general. On the contrary, the gauge theory has no analogue of vielbeins or a Riemannian metric and it is described by a quadratic action of curvature tensors. Consequently this difference brings about a different scaling behavior of coupling constants in gravity and gauge theory, which causes the notorious problem for the renormalization of gravity.

Above all, the gauge theory formulation of gravity may uncover a profound reason why gravity enjoys a peculiar structure apart from the homomorphy with gauge theory. Therefore, in order to appreciate an origin of the contrast between gravity and gauge theory, we will undertake a systematic formulation of Einstein gravity as a gauge theory of local Lorentz symmetry and answer

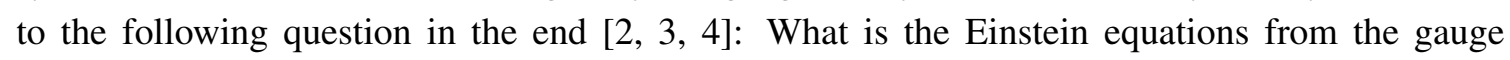
theory point of view? Or equivalently, what is the gauge theory object corresponding to Einstein manifolds?

\section{Gauge Theory of Riemannian Manifolds}

Let $M$ be a $d$-dimensional Riemannian manifold $M$ whose metric is given by

$$
d s^{2}=g_{M N}(x) d x^{M} \otimes d x^{N}, \quad M, N=1, \cdots, d
$$

and introduce at each spacetime point on $M$ a local basis of orthonormal tangent vectors (vielbeins) $E_{A}=E_{A}^{M} \partial_{M} \in \Gamma(T M), A=1, \cdots, d$. The frame basis $\left\{E_{A}\right\}$ defines a dual basis $E^{A}=E_{M}^{A} d x^{M} \in$ 
$\Gamma\left(T^{*} M\right)$ by a natural pairing $\left\langle E^{A}, E_{B}\right\rangle=\delta_{B}^{A}$. In terms of the non-coordinate (anholonomic) basis in $\Gamma(T M)$ or $\Gamma\left(T^{*} M\right)$, the metric (2. $($ ) can be written as

$$
d s^{2}=\delta_{A B} E^{A} \otimes E^{B}=\delta_{A B} E_{M}^{A} E_{N}^{B} d x^{M} \otimes d x^{N}=g_{M N}(x) d x^{M} \otimes d x^{N}
$$

or

$$
\left(\frac{\partial}{\partial s}\right)^{2}=\delta^{A B} E_{A} \otimes E_{B}=\delta^{A B} E_{A}^{M} E_{B}^{N} \partial_{M} \otimes \partial_{N}=g^{M N}(x) \partial_{M} \otimes \partial_{N}
$$

There is a large arbitrariness in the choice of a vielbein because the vielbein formalism respects a local gauge invariance. Under a local Lorentz transformation which is an orthogonal frame rotation in $S O(d)$, the basis vectors transform according to

$$
\begin{aligned}
& E_{A}(x) \rightarrow E_{A}^{\prime}(x)=E_{B}(x) \Lambda^{B}{ }_{A}(x), \\
& E^{A}(x) \rightarrow E^{A^{\prime}}(x)=\Lambda^{A}{ }_{B}(x) E^{B}(x)
\end{aligned}
$$

where $\Lambda_{B}^{A}(x) \in S O(d)$ is a local Lorentz transformation. As in any other discussion of local gauge invariance, to achieve the local Lorentz invariance requires introducing a gauge field. On a Riemannian manifold $M$, the spin connection $\omega$ is an $S O(d)$ gauge field [四]. To be precise, a matrix-valued spin connection $\omega=\frac{1}{2} \omega_{A B} J^{A B}=\frac{1}{2} \omega_{M A B}(x) J^{A B} d x^{M}$ constitutes a gauge field with respect to the local $S O(d)$ rotations

$$
\omega_{M} \rightarrow \omega_{M}^{\prime}=\Lambda \omega_{M} \Lambda^{-1}+\Lambda \partial_{M} \Lambda^{-1}
$$

where $\Lambda=\exp \left(\frac{1}{2} \lambda_{A B}(x) J^{A B}\right) \in S O(d)$ and $J^{A B}$ are $S O(d)$ Lorentz generators which satisfy the following Lorentz algebra

$$
\left[J^{A B}, J^{C D}\right]=-\left(\delta^{A C} J^{B D}-\delta^{A D} J^{B C}-\delta^{B C} J^{A D}+\delta^{B D} J^{A C}\right) .
$$

Then the covariant derivatives for the vectors in Eq. (R.4) are defined by

$$
\begin{aligned}
& D_{M} E_{A}=\partial_{M} E_{A}-\omega_{M}{ }^{B} E_{B}, \\
& D_{M} E^{A}=\partial_{M} E^{A}+\omega_{M}{ }_{B}^{A} E^{B} .
\end{aligned}
$$

The connection one-forms $\omega^{A}{ }_{B}=\omega_{M}{ }_{B}{ }_{B} d x^{M}$ satisfy the Cartan's structure equations [四, 回]

$$
\begin{aligned}
T^{A} & =d E^{A}+\omega^{A}{ }_{B} \wedge E^{B}, \\
R_{B}^{A} & =d \omega^{A}{ }_{B}+\omega^{A}{ }_{C} \wedge \omega^{C}{ }_{B},
\end{aligned}
$$

where $T^{A}$ are the torsion two-forms and $R_{B}^{A}$ are the curvature two-forms. We impose the torsion free condition, $T_{M N}{ }^{A}=D_{M} E_{N}^{A}-D_{N} E_{M}^{A}=0$, to recover the standard content of general relativity, which eliminates $\omega_{M}$ as an independent variable, i.e.,

$$
\omega_{A B C}=E_{A}^{M} \omega_{M B C}=\frac{1}{2}\left(f_{A B C}-f_{B C A}+f_{C A B}\right)
$$

where $f_{A B C}$ are the structure functions defined by

$$
\left[E_{A}, E_{B}\right]=-f_{A B}^{C} E_{C} .
$$


For orthogonal groups the second-rank antisymmetric tensor representation is the same as the adjoint representation, so the Lorentz generators $J^{A B}=-J^{B A}, A, B=1, \cdots, d$, can be conveniently labeled as $T^{a}, a=1, \cdots, \frac{d(d-1)}{2}$. Hence, we now introduce an $S O(d)$-valued gauge field defined by $A=A^{a} T^{a}$ where $A^{a}=A_{M}^{a} d x^{M}$ are connection one-forms on $M$ and $T^{a}$ are Lie algebra generators of $S O(d)$ satisfying

$$
\left[T^{a}, T^{b}\right]=-f^{a b c} T^{c}
$$

In order to formulate the gauge theory of gravity, it will be useful to adopt the following local isomorphism for $d$-dimensional Lorentz group $S O(d)$ :

$$
S O(3) \cong S U(2), \quad S O(4) \cong S U(2)_{L} \times S U(2)_{R}, \quad S O(5) \cong S p(2), \quad S O(6) \cong S U(4) .
$$

Another useful relation is the isomorphism between the Clifford algebra $\mathbb{C l}(d)$ in $d$-dimensions and the exterior algebra $\Lambda^{*} M$ of cotangent bundle $T^{*} M$ over a $d$-dimensional Riemannian manifold $M$ [焑]:

$$
\mathbb{C} l(d) \cong \Lambda^{*} M=\bigoplus_{k=0}^{d} \Lambda^{k} T^{*} M .
$$

In this correspondence, the chiral operator $\Gamma^{d+1}$ in even dimensions corresponds to the Hodge star operation $*: \Lambda^{k} T^{*} M \rightarrow \Lambda^{d-k} T^{*} M$ in $\Lambda^{*} M$. In particular, the Clifford map (2.14) implies that the $S O(d)$ Lorentz generators $J^{A B}=\frac{1}{4}\left[\Gamma^{A}, \Gamma^{B}\right]$ in $\mathbb{C l}(d)$ have one-to-one correspondence with the space $\Lambda^{2} T^{*} M$ of two-forms in $\Lambda^{*} M$ where $\Gamma^{A}$ are $d$-dimensional Dirac matrices obeying the Dirac algebra

$$
\left\{\Gamma^{A}, \Gamma^{B}\right\}=2 \delta^{A B} \mathbf{I}_{2^{\left[\frac{d}{2}\right]}} .
$$

The identification [0, 团] we want to make is then given by ${ }^{1}$

$$
\omega=\frac{1}{2} \omega_{A B} J^{A B} \equiv A=A^{a} T^{a} .
$$

In particular, the isomorphism (2.13) for four-dimensional Lorentz group $S O(4)$ suggests that the spin connections in four dimensions are split into a pair of $S U(2)_{L}$ and $S U(2)_{R}$ gauge fields. The Lorentz transformation (2.5]) can then be translated into a usual gauge transformation

$$
A \rightarrow A^{\prime}=\Lambda A \Lambda^{-1}+\Lambda d \Lambda^{-1}
$$

where $\Lambda=e^{\lambda^{a}(x) T^{a}} \in S O(d)$. The $S O(d)$-valued Riemann curvature tensor is defined by

$$
\begin{aligned}
R & =d \omega+\omega \wedge \omega \\
& =\frac{1}{2} R_{A B} J^{A B}=\frac{1}{2}\left(d \omega_{A B}+\omega_{A C} \wedge \omega_{C B}\right) J^{A B} \\
& =\frac{1}{4}\left[\left(\partial_{M} \omega_{N A B}-\partial_{N} \omega_{M A B}+\omega_{M A C} \omega_{N C B}-\omega_{N A C} \omega_{M C B}\right) J^{A B}\right] d x^{M} \wedge d x^{N}
\end{aligned}
$$

\footnotetext{
${ }^{1}$ To be precise, the spin connection (D.J6) is a connection on a spinor bundle induced from the $S O(d)$-bundle and the structure group of its fiber is lifted to $\operatorname{Spin}(d)$, a double cover of $S O(d)$, according to the short exact sequence of Lie groups: $1 \rightarrow \mathbb{Z}_{2} \rightarrow \operatorname{Spin}(d) \rightarrow S O(d) \rightarrow 1$. Hence the global isomorphism should refer to $\operatorname{Spin}(d)$. Nevertheless we will not care about the $\mathbb{Z}_{2}$-factor because we are mostly interested in local descriptions (in the level of Lie algebras) and we will not be careful to discriminate a Lie group and its Lie algebra.
} 
or, in terms of gauge theory variables, it is given by

$$
\begin{aligned}
F & =d A+A \wedge A \\
& =F^{a} T^{a}=\left(d A^{a}-\frac{1}{2} f^{a b c} A^{b} \wedge A^{c}\right) T^{a} \\
& =\frac{1}{2}\left[\left(\partial_{M} A_{N}^{a}-\partial_{N} A_{M}^{a}-f^{a b c} A_{M}^{b} A_{N}^{c}\right) T^{a}\right] d x^{M} \wedge d x^{N} .
\end{aligned}
$$

Using the form language where $d=d x^{M} \partial_{M}=E^{A} E_{A}$ and $A=A_{M} d x^{M}=A_{A} E^{A}$, the field strength ([.19) of $S O(d)$ gauge fields in the non-coordinate basis takes the form

$$
\begin{aligned}
F & =d A+A \wedge A=\frac{1}{2} F_{A B} E^{A} \wedge E^{B} \\
& =\frac{1}{2}\left(E_{A} A_{B}-E_{B} A_{A}+\left[A_{A}, A_{B}\right]+f_{A B}{ }^{C} A_{C}\right) E^{A} \wedge E^{B}
\end{aligned}
$$

where we used the structure equation

$$
d E^{A}=\frac{1}{2} f_{B C}{ }^{A} E^{B} \wedge E^{C} .
$$

Then it is easy to find the gauge theory representation for the second Bianchi identity for Riemann curvature tensors which is mapped to the Bianchi identity for Yang-Mills field strengths [2]], i.e.,

$$
D R \equiv d R+\omega \wedge R-R \wedge \omega=0 \quad \Leftrightarrow \quad D F \equiv d F+A \wedge F-F \wedge A=0 .
$$

\section{Four-Manifolds and Yang-Mills Instantons}

The four dimensional space has mystic features. Among the group of isometries of $d$-dimensional Euclidean space $\mathbb{R}^{d}$, the Lie group $S O(4)$ for $d \geq 3$ is the only non-simple Lorentz group as was shown in (2.13) and one can define a self-dual two-form only for $d=4$. If $M$ is an oriented fourmanifold, the Hodge *-operation defines an automorphism of the vector space $\Lambda^{2} T^{*} M$ of two-forms with the decomposition

$$
\Lambda^{2} T^{*} M=\Lambda_{3}^{+} \oplus \Lambda_{3}^{-}
$$

where $\Lambda_{3}^{ \pm} \equiv P_{ \pm} \Lambda^{2} T^{*} M$ and $P_{ \pm}=\frac{1}{2}(1 \pm *)$. That is, in four dimensions, the six-dimensional vector space $\Lambda^{2} T^{*} M$ of two-forms splits canonically into the sum of three-dimensional vector spaces of self-dual and anti-self-dual two forms [ [ $[$, [8]. In order to answer to the previous question, it is enough to note such a plain fact that the Lorentz group $S O(4)$ is isomorphic to $S U(2)_{L} \times S U(2)_{R}$ and the Riemann curvature tensor $R_{B}^{A}=d \omega^{A}{ }_{B}+\omega^{A}{ }_{C} \wedge \omega^{C}{ }_{B}$ is an $S O(4)$-valued two-form. One can thus apply two kinds of decomposition to spin connections and curvature tensors. The first decomposition is that the spin connections $\omega^{A}{ }_{B}$ can be split into a pair of $S U(2)_{L}$ and $S U(2)_{R}$ gauge fields according to the splitting of the Lie algebra $S O(4)=S U(2)_{L} \oplus S U(2)_{R}$ [, 团]:

$$
\omega_{M A B} \equiv A_{M}^{(+) a} \eta_{A B}^{a}+A_{M}^{(-) \dot{a}} \bar{\eta}_{A B}^{\dot{a}}
$$

where $A^{(+) a}=A_{M}^{(+) a} d x^{M}$ and $A^{(-) \dot{a}}=A_{M}^{(-) \dot{a}} d x^{M}$ are $S U(2)_{L}$ and $S U(2)_{R}$ gauge fields, respectively. Accordingly the Riemann curvature tensor $R_{B}^{A}$ will also be decomposed into a pair of $S U(2)_{L}$ and $S U(2)_{R}$ curvature two-forms:

$$
R_{M N A B} \equiv F_{M N}^{(+) a} \eta_{A B}^{a}+F_{M N}^{(-) \dot{a}} \bar{\eta}_{A B}^{\dot{a}}
$$


where

$$
F_{M N}^{( \pm)}=\partial_{M} A_{N}^{( \pm)}-\partial_{N} A_{M}^{( \pm)}+\left[A_{M}^{( \pm)}, A_{N}^{( \pm)}\right]
$$

are field strengths of $S U(2)_{L}$ and $S U(2)_{R}$ gauge fields in (B.2).

The second decomposition is to apply the Hodge $*$-decomposition (B.D) to the Riemann curvature tensors in (B.3) $)$. Because $S U(2)_{L}$ and $S U(2)_{R}$ field strengths defined by $F^{(+) a}=\frac{1}{2} F_{M N}^{(+) a} d x^{M} \wedge$ $d x^{N}$ and $F^{(-) \dot{a}}=\frac{1}{2} F_{M N}^{(-) \dot{a}} d x^{M} \wedge d x^{N}$, respectively, are curvature two-forms in gauge theory, both of them can be split into a pair of self-dual and anti-self-dual two-forms as follows

$$
\begin{aligned}
F_{A B}^{(+) a} & \equiv f_{(++)}^{a b} \eta_{A B}^{b}+f_{(+-)}^{a \dot{b}} \bar{\eta}_{A B}^{\dot{b}}, \\
F_{A B}^{(-) \dot{a}} & \equiv f_{(-+)}^{\dot{a} b} \eta_{A B}^{b}+f_{(--)}^{\dot{a} \dot{b}} \bar{\eta}_{A B}^{\dot{b}} .
\end{aligned}
$$

Using the above result, we get the following decomposition of the Riemann curvature tensor [B]

$$
R_{A B C D}=f_{(++)}^{a b} \eta_{A B}^{a} \eta_{C D}^{b}+f_{(+-)}^{a \dot{b}} \eta_{A B}^{a} \bar{\eta}_{C D}^{\dot{b}}+f_{(-+)}^{\dot{a} b} \bar{\eta}_{A B}^{\dot{a}} \eta_{C D}^{b}+f_{(--)}^{\dot{a} \dot{b}} \bar{\eta}_{A B}^{\dot{a}} \bar{\eta}_{C D}^{\dot{b}}
$$

The first Bianchi identity $\varepsilon^{A C D E} R_{B C D E}=0$ constrains the coefficients in the expansion (B.7) which reads as

$$
f_{(++)}^{a b}=f_{(++)}^{b a}, \quad f_{(--)}^{\dot{a} \dot{b}}=f_{(--)}^{\dot{b} \dot{a}}, \quad f_{(+-)}^{a \dot{b}}=f_{(-+)}^{\dot{b} a}
$$

and

$$
f_{(++)}^{a b} \delta^{a b}=f_{(--)}^{\dot{a} \dot{b}} \delta^{\dot{a} \dot{b}} .
$$

Now we can state the Lemma for general Einstein manifolds proven in [B], 团].

Lemma. The Riemann curvature two-form $R_{A B}=\frac{1}{2} R_{M N A B} d x^{M} \wedge d x^{N}$ is an $S O(4)$-valued field strength of the spin connections in (B.2) from the gauge theory point of view and thus can be decomposed into a pair of $S U(2)_{L}$ and $S U(2)_{R}$ field strengths. With the decomposition (B.3)), the Einstein equation

$$
R_{A B}-\frac{1}{2} \delta_{A B} R+\delta_{A B} \Lambda=0
$$

for a Riemannian manifold $M$ is equivalent to the self-duality equation of Yang-Mills instantons

$$
F_{A B}^{( \pm)}= \pm \frac{1}{2} \varepsilon_{A B}^{C D} F_{C D}^{( \pm)}
$$

where $F_{A B}^{(+) a} \eta_{A B}^{a}=F_{A B}^{(-) \dot{a}} \bar{\eta}_{A B}^{\dot{a}}=2 \Lambda$.

One can draw a very interesting implication [1], 团] from the above lemma. The $S U(2)$ field strengths in Eq. ([.3.]) are given by

$$
F^{( \pm)}=d A^{( \pm)}+A^{( \pm)} \wedge A^{( \pm)}
$$

The integrability condition, namely, the Bianchi identity, then reads as

$$
D^{( \pm)} F^{( \pm)} \equiv d F^{( \pm)}+A^{( \pm)} \wedge F^{( \pm)}-F^{( \pm)} \wedge A^{( \pm)}=0 .
$$

Therefore the self-duality equation (B.DI) immediately leads to the remarkable result that any Einstein manifold automatically satisfies the Yang-Mills equations of motion, i.e.,

$$
D^{( \pm)} * F^{( \pm)}= \pm D^{( \pm)} F^{( \pm)}=0 \Leftrightarrow D * F=D^{(+)} * F^{(+)}+D^{(-)} * F^{(-)}=0
$$


where $* F$ means the Hodge $*$-operation on a two-form $F$. After all, our lemma sheds light on why the action of Einstein gravity is linear in curvature tensors contrary to the Yang-Mills action being quadratic in curvatures. If the action of Einstein gravity were quadratic in curvature tensors, fourmanifolds obeying the equations of motion would not necessarily be given by $S U$ (2) Yang-Mills instantons and the four-manifold could be unstable in general as is well-known from gauge theory.

\section{Topology of Four-Manifolds}

The topological invariants for four-manifolds have a local expression due to the Atiyah-Singer index theorem [ [5, 四]. For a general closed manifold $M$, the Euler number $\chi(M)$ for the de Rham complex and the signature $\tau(M)$ for the Hirzebruch signature complex are defined by

$$
\begin{aligned}
& \chi(M)=-\frac{1}{8 \pi^{2}} \int_{M} \operatorname{Tr} \Gamma^{5} R \wedge R=\frac{1}{32 \pi^{2}} \int_{M} \varepsilon^{A B C D} R_{A B} \wedge R_{C D}, \\
& \tau(M)=-\frac{1}{12 \pi^{2}} \int_{M} \operatorname{Tr} R \wedge R=\frac{1}{24 \pi^{2}} \int_{M} R_{A B} \wedge R_{A B} .
\end{aligned}
$$

It is then straightforward to express the topological invariants with $S U(2)$ gauge fields using the decomposition (3.7) [B]

$$
\begin{aligned}
\chi(M) & =\frac{1}{4 \pi^{2}} \int_{M}\left(F^{(+) a} \wedge F^{(+) a}-F^{(-) \dot{a}} \wedge F^{(-) \dot{a}}\right) \\
& =\frac{1}{2 \pi^{2}} \int_{M} d^{4} x \sqrt{g}\left[\left(f_{(++)}^{a b}\right)^{2}+\left(f_{(--)}^{\dot{a} \dot{b}}\right)^{2}-2\left(f_{(+-)}^{a \dot{b}}\right)^{2}\right] \\
\tau(M) & =\frac{1}{6 \pi^{2}} \int_{M}\left(F^{(+) a} \wedge F^{(+) a}+F^{(-) \dot{a}} \wedge F^{(-) \dot{a}}\right) \\
& =\frac{1}{3 \pi^{2}} \int_{M} d^{4} x \sqrt{g}\left[\left(f_{(++)}^{a b}\right)^{2}-\left(f_{(--)}^{\dot{a} \dot{b}}\right)^{2}\right] .
\end{aligned}
$$

Since Einstein manifolds carry a topological information in the form of Yang-Mills instantons as was shown before, it will be interesting to see how the topology of spacetime fabric is encoded into gauge fields. An Einstein manifold has a curvature tensor given by (B.7) with the coefficients satisfying $f_{(+-)}^{a \dot{b}}=f_{(-+)}^{\dot{b} a}=0$ [B]]. In this case, the Euler characteristic $\chi(M)$ is given by the sum of self-dual and anti-self-dual instantons whereas the signature $\tau(M)$ is their difference. Then Eq. (4.3) immediately shows $\chi(M) \geq 0$ with equality only if $f_{(++)}^{a b}=f_{(--)}^{\dot{a} \dot{b}}=0$, i.e., $M$ is flat. Moreover, it is easy to get the Hitchin-Thorpe inequality [1], [0]

$$
\chi(M) \pm \frac{3}{2} \tau(M)=\frac{1}{\pi^{2}} \int_{M} d^{4} x \sqrt{g}\left(f_{( \pm \pm)}^{\dot{a} \dot{b}}\right)^{2} \equiv 2 \chi_{ \pm}(M) \geq 0
$$

where the equality holds if and only if $f_{( \pm \pm)}^{\dot{a} \dot{b}}=0$, i.e., $M$ is half-flat (a gravitational instanton).

After all, the topology of Einstein manifolds is characterized by intanton and anti-instanton configurations. Let

$$
\begin{aligned}
& \chi(M)=\chi_{+}(M)+\chi_{-}(M) \equiv m \in \mathbb{Z}_{\geq 0}, \\
& \tau(M)=\frac{2}{3}\left(\chi_{+}(M)-\chi_{-}(M)\right) \equiv n \in \mathbb{Z}
\end{aligned}
$$




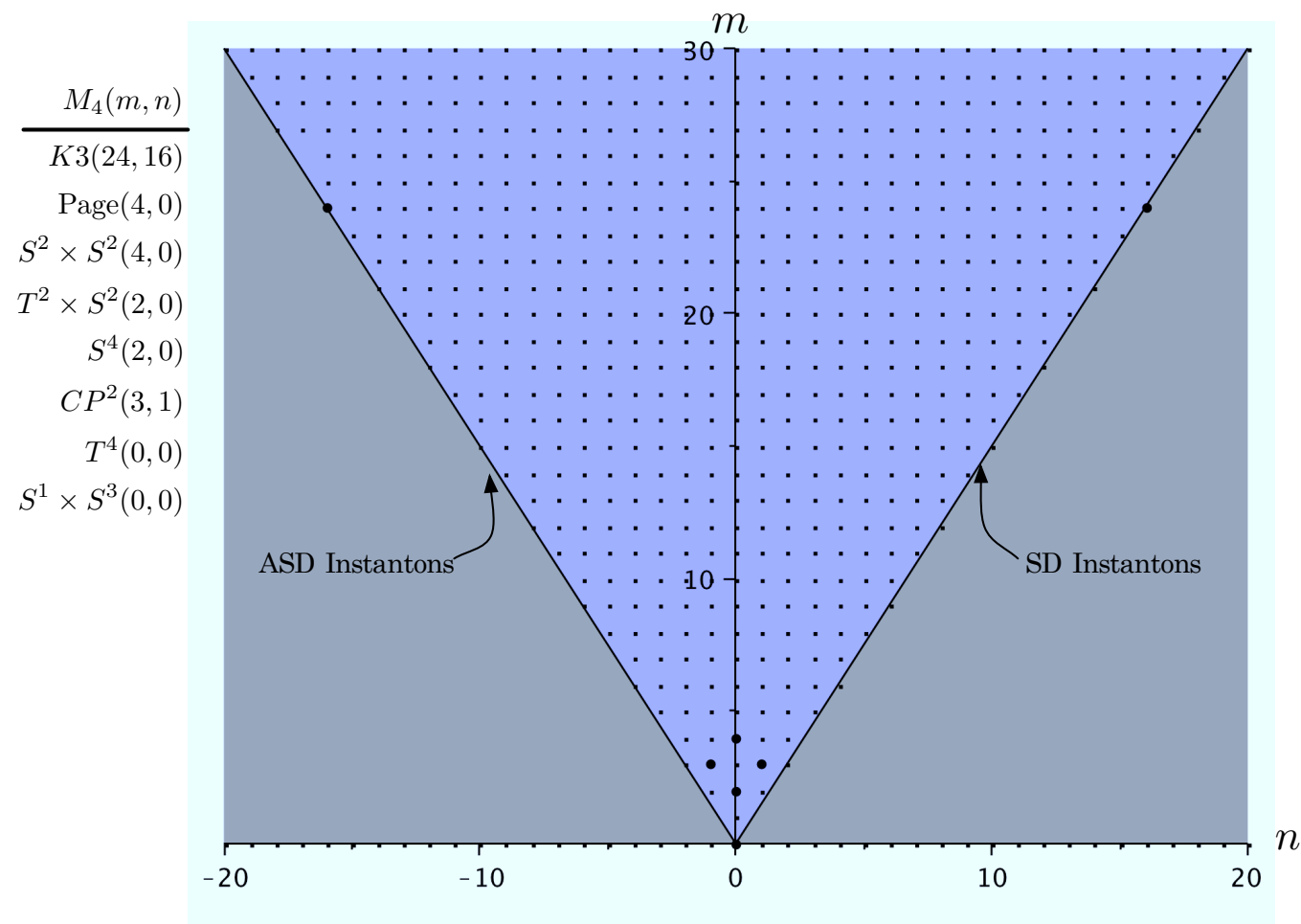

Figure 1: Topological numbers of closed Einstein manifolds

In four dimensions, we can define an intersection form $Q: H^{2} \times H^{2} \rightarrow \mathbb{R}$ by [ [ $]$

$$
Q(\alpha, \beta)=\int_{M} \alpha \wedge \beta
$$

where $H^{2}:=H^{2}(M, \mathbb{R})$ is the second de Rham cohomology and $\alpha, \beta \in H^{2}(M, \mathbb{R})$. The decomposition (B. $\mathrm{C}$ ) can descend to the de Rham cohomology and so we find a similar decomposition $H^{2}=H_{+}^{2} \oplus H_{-}^{2}$. The Betti number $b_{k}=\operatorname{dim} H^{k}(M, \mathbb{R})$ of $M$ is the dimension of $k$-th cohomology group and so we have $b_{2}=b_{2}^{+}+b_{2}^{-}$with

$$
b_{2}^{ \pm}=\operatorname{dim} H_{ \pm}^{2}
$$

The signature $\tau(M)$ is then defined as the difference of positive and negative eigenvalues of $Q$

$$
\tau(M)=b_{2}^{+}-b_{2}^{-} .
$$

The Euler characteristic $\chi(M)$ is of course defined by $\chi(M)=\sum_{k=0}^{4}(-1)^{k} b_{k}=2-2 b_{1}+b_{2}$, which results from the Poincaré duality and may be expressed as $\chi(M)=b_{2} \bmod 2$. So one can deduce that $\chi+\tau=2\left(1-b_{1}+b_{2}^{+}\right) \in 2 \mathbb{Z}[\square,[]]$. Hence we can conclude that the set $(m, n)$ of topological numbers forms an even integer lattice, i.e., $m+n \in 2 \mathbb{Z}$. Furthermore, one can get the inequality $2 m \pm 3 n \geq 0$ from Eq. (4.5). Consequently any closed Einstein manifold should appear in upper wedge of the even integer lattice $(m, n)$ as shown in Fig. 1.

The Figure 1 reveals some interesting features. First suppose that the first Betti number is zero, $b_{1}=0$, and so $b_{2}^{ \pm}+1=\frac{1}{2}(m \pm n)$. In this case the inequality $b_{2}^{ \pm}+1 \geq \frac{3 \pm 2}{6} m$ holds where the 
equality occurs when $M$ is a gravitational instanton, i.e., $F^{( \pm)}=0$ or equivalently $m=\frac{3}{2}|n|$. For a $\mathrm{K} 3$ surface, for example, $m=24, b_{2}^{+}=19$ and $b_{2}^{-}=3[\square]$. Another intriguing aspect of the figure is that it shows a left-right reflection symmetry. In order to examine the "reflection" symmetry, let us consider two compact Einstein manifolds $(M, g)$ and $(\widetilde{M}, \widetilde{g})$ obeying the following relation

$$
\chi_{+}(M)=\chi_{-}(\widetilde{M})=\frac{2 m+3 n}{4}, \quad \chi_{-}(M)=\chi_{+}(\widetilde{M})=\frac{2 m-3 n}{4} .
$$

Under the above transformation (4.]), the topological invariants are related as follows

$$
\chi(M)=\chi(\widetilde{M}) \geq 0, \quad \tau(M)=-\tau(\widetilde{M}) .
$$

Thereby we can rephrase the reflection map (4.JI) as an interchange of the signature $\left(b_{2}^{+}, b_{2}^{-}\right)$of the intersection form $Q$ in Eq. (4.8):

$$
b_{2}^{+}(M)=b_{2}^{-}(\widetilde{M}), \quad b_{2}^{-}(M)=b_{2}^{+}(\widetilde{M}) .
$$

This map indicates that a four-manifold with $b_{2}^{+}=b_{2}^{-}$, i.e. $\tau(M)=0$, will be self-mirror.

\section{Calabi-Yau Manifolds and Hermitian Yang-Mills Instantons}

We now apply the gauge theory formulation to six-dimensional Riemannian manifolds [9]. For this purpose, $S O(6)$ Lorentz group for Euclidean gravity will be identified with $S U(4)$ gauge group in Yang-Mills gauge theory according to the isomorphism (2.13). Via the gauge theory formulation of six-dimensional Euclidean gravity, we want to find a gauge theory object corresponding to Calabi-Yau manifolds.

Let us start with the Clifford algebra $\mathbb{C l}(6)$ whose generators are given by

$$
\mathbb{C l}(6)=\left\{\mathbf{I}_{8}, \Gamma^{A}, \Gamma^{A B}, \Gamma_{ \pm}^{A B C}, \Gamma^{7} \Gamma^{A B}, \Gamma^{7} \Gamma^{A}, \Gamma^{7}\right\}
$$

where $\Gamma^{A}(A=1, \cdots, 6)$ are six-dimensional Dirac matrices satisfying the algebra ([2.5]), $\Gamma^{A_{1} A_{2} \cdots A_{k}}=$ $\frac{1}{k !} \Gamma^{\left[A_{1}\right.} \Gamma^{A_{2}} \cdots \Gamma_{k}^{\left.A_{k}\right]}$ with the complete antisymmetrization of indices, the chiral matrix $\Gamma^{7}=i \Gamma^{1} \cdots \Gamma^{6}$ and $\Gamma_{ \pm}^{A B C}=\frac{1}{2}\left(\mathbf{I}_{8} \pm \Gamma^{7}\right) \Gamma^{A B C}$. The Clifford map ([2.14) suggests that the Clifford algebra (1. be identified with the exterior algebra $\Lambda^{*} M=\bigoplus_{k=0}^{6} \Lambda^{k} T^{*} M$.

The spinor representation of the algebra (1) has two irreducible spinor representations $S_{ \pm}$ each of dimension 4, namely the spinors of positive and negative chirality, i.e. $V=S_{+} \oplus S_{-}$, where the Hilbert space $V$ of dimension 8 is defined by

$$
V=\bigoplus_{k=0}^{3}\left|\Omega_{i_{1} \cdots i_{k}}\right\rangle=\bigoplus_{k=0}^{3} a_{i_{1}}^{*} \cdots a_{i_{k}}^{*}|\Omega\rangle .
$$

If the Fock vacuum $|\Omega\rangle$ has positive chirality, the positive chirality spinors are states given by

$$
S_{+}=\bigoplus_{k \text { even }}\left|\Omega_{i_{1} \cdots i_{k}}\right\rangle=|\Omega\rangle+\left|\Omega_{i j}\right\rangle=\mathbf{4}
$$

while the negative chirality spinors are those obtained by

$$
S_{-}=\bigoplus_{k \text { odd }}\left|\Omega_{i_{1} \cdots i_{k}}\right\rangle=\left|\Omega_{i}\right\rangle+\left|\Omega_{i j k}\right\rangle=\overline{\mathbf{4}} .
$$


As the $S O(6)$ Lorentz algebra is isomorphic to the Lie algebra of $S U(4)$, the positive- and negativechirality spinors of $S O(6)$ can be identified with the fundamental representation 4 and the antifundamental representation $\overline{4}$ of $S U(4)$, respectively [प]]].

One can form a direct product of the fundamental representations $\mathbf{4}$ and $\overline{4}$ of $S U$ (4) in order to classify the Clifford generators in Eq. (D.]

$$
\begin{aligned}
& \mathbf{4} \otimes \overline{\mathbf{4}}=\mathbf{1} \oplus \mathbf{1 5}=\left\{\Gamma_{+}, \Gamma_{+}^{A B}\right\}, \\
& \overline{\mathbf{4}} \otimes \mathbf{4}=\mathbf{1} \oplus \mathbf{1 5}=\left\{\Gamma_{-}, \Gamma_{-}^{A B}\right\}, \\
& \mathbf{4} \otimes \mathbf{4}=\mathbf{6} \oplus \mathbf{1 0}=\left\{\Gamma_{+}^{A}, \Gamma_{+}^{A B C}\right\}, \\
& \overline{\mathbf{4}} \otimes \overline{\mathbf{4}}=\mathbf{6} \oplus \mathbf{1 0}=\left\{\Gamma_{-}^{A}, \Gamma_{-}^{A B C}\right\},
\end{aligned}
$$

where $\Gamma_{ \pm} \equiv \frac{1}{2}\left(\mathbf{I}_{8} \pm \Gamma^{7}\right), \Gamma_{ \pm}^{A} \equiv \Gamma_{ \pm} \Gamma^{A}$ and $\Gamma_{ \pm}^{A B} \equiv \Gamma_{ \pm} \Gamma^{A B}$. Note that $\mathbf{1 5}$ in (5.5) and (5.6) is the adjoint representation of $S U(4)$ and $\mathbf{6}$ in (5.7) and (5.8) is the antisymmetric second-rank tensor of $S U$ (4) while $\mathbf{1 0}$ is the symmetric second-rank tensor of $S U(4)$. It is worthwhile to remark that $\Gamma_{ \pm}^{2}=\Gamma_{ \pm}, \Gamma_{+} \Gamma_{-}=0$ and so $\Gamma_{ \pm}$is a projection operator onto the space of definite chirality.

A new feature different from the four-dimensional case is that there are two sources of twoforms. One is from two-forms themselves in $\Lambda^{2} T^{*} M$ and the other is from the Hodge-dual of fourforms in $\Lambda^{4} T^{*} M$. This duplication of two-forms results in the doubling of two-form basis, i.e., $\Lambda^{2} T^{*} M \oplus *\left(\Lambda^{4} T^{*} M\right)$. Also note that $\Gamma_{+}^{A B} \in \mathbf{1 5}$ in (5.5) and $\Gamma_{-}^{A B} \in \mathbf{1 5}$ in (5.6) are independent of each other as we remarked above and this doubling of the Clifford basis is parallel to the doubling of two-forms according to the Clifford map (2.14). Hence $\Gamma_{+}^{A B} \in \mathbf{1 5}$ and $\Gamma_{-}^{A B} \in \mathbf{1 5}$ will serve as independent bases of two-forms.

According to the identification (R.16), we have the following relation [Q]

$$
R_{A B}=\frac{1}{2} R_{A B C D} J^{C D}=\left(F_{A B}^{(+) a} T_{1}^{a} \oplus F_{A B}^{(-) a} T_{2}^{a}\right)=F_{A B},
$$

where $T_{1}^{a}$ and $T_{2}^{a}$ are $S U(4)$ Lie algebra generators defined by (‥12). On the right hand side, the doubling of $S U(4)$ algebra in four-dimensional representations $R_{1}$ and $R_{2}$ was considered because the $S O(6)$ spinor representation on the left hand side is eight-dimensional and reducible. Thus $R_{A B}$ on the left hand side has twice as many components as the usual Riemann curvature tensor. But, as we already pointed out above, the $S U(4)$-valued two-form $R_{A B}$ must be regarded as a mixture of two-forms in $\Lambda^{2} T^{*} M$ and the Hodge-dual of four-forms in $\Lambda^{4} T^{*} M .^{2}$ As already indicated by (5.9), this mixture of $\Lambda^{2} T^{*} M \oplus *\left(\Lambda^{4} T^{*} M\right)$ has an irreducible decomposition as follows. We will regard the Riemann tensor $R_{A B}$ as a linear map acting on the Hilbert space $V$ in Eq. (5.2). As $R_{A B}$ contains two gamma matrices, it does not change the chirality of the vector space $V$. Therefore, we can represent it in a subspace of definite chirality as either $R_{A B}: S_{+} \rightarrow S_{+}$or $R_{A B}: S_{-} \rightarrow S_{-}$. The former case $R_{A B}: S_{+} \rightarrow S_{+}$will take values in $\mathbf{4} \otimes \overline{\mathbf{4}}$ in (5.5) with a singlet being removed while the latter case $R_{A B}: S_{-} \rightarrow S_{-}$will take values in $\overline{\mathbf{4}} \otimes \mathbf{4}$ in (5.6) with no singlet.

Therefore, there exist two independent identifications defined by

$$
\begin{aligned}
& \mathbb{A}: \frac{1}{2} R_{A B C D} J_{+}^{C D} \equiv F_{A B}^{(+) a}\left(T^{a} \oplus \mathbf{0}\right), \\
& \mathbb{B}: \frac{1}{2} R_{A B C D} J_{-}^{C D} \equiv F_{A B}^{(-) a}\left(\mathbf{0} \oplus T^{a}\right),
\end{aligned}
$$

\footnotetext{
${ }^{2}$ In other words, we think of $R_{A B}$ to contain the usual curvature tensor in (R.g) as well as another curvature tensor defined by $\widetilde{R}_{A B} \equiv * G_{A B}=d \widetilde{\omega}_{A B}+\widetilde{\omega}_{A C} \wedge \widetilde{\omega}_{C B}$ where $G_{A B}$ is an $S O(6)$-valued form-form tensor.
} 
where we distinguish the two classes $\mathbb{A}$ and $\mathbb{B}$ depending on the six-dimensional chirality. Because the classes $\mathbb{A}$ and $\mathbb{B}$ are now represented by $4 \times 4$ matrices on both sides, we can take a trace operation for the matrices which leads to the following relations

$$
\begin{aligned}
& \mathbb{A}: R_{A B C D}=-F_{A B}^{(+) a} \operatorname{Tr}\left(T^{a} J_{+}^{C D}\right) \equiv F_{A B}^{(+) a} \eta_{C D}^{a} \\
& \mathbb{B}: R_{A B C D}=-F_{A B}^{(-) a} \operatorname{Tr}\left(T^{a} J_{-}^{C D}\right) \equiv F_{A B}^{(-) a} \bar{\eta}_{C D}^{a} .
\end{aligned}
$$

Here we have introduced the six-dimensional analogue of the 't Hooft symbols defined by

$$
\eta_{A B}^{a}=-\operatorname{Tr}\left(T^{a} J_{+}^{A B}\right), \quad \bar{\eta}_{A B}^{a}=-\operatorname{Tr}\left(T^{a} J_{-}^{A B}\right) .
$$

An explicit representation of the six-dimensional 't Hooft symbols can be found in [Q $\mathrm{Q}]$. Similarly, $S O(6)$ spin connection $\omega=\frac{1}{2} \omega_{A B} J^{A B}$ can be identified with (doubled) $S U(4)$ gauge fields. From the identification (2.16), we get the following relation

$$
\omega^{( \pm)} \equiv \Gamma_{ \pm} \omega=A^{( \pm) a} T^{a}, \quad A^{( \pm) a}=-2 \operatorname{Tr}\left(T^{a} \omega^{( \pm)}\right) .
$$

The corresponding $S U(4)$ field strengths are defined by (R.T) and appear in the chiral sectors in (5.12) and (5.13)

Consider an arbitrary two-form

$$
F=\frac{1}{2} F_{M N} d x^{M} \wedge d x^{N}=\frac{1}{2} F_{A B} E^{A} \wedge E^{B} \in \Lambda^{2} T^{*} M
$$

and introduce 15-dimensional basis of two-forms in $\Lambda^{2} T^{*} M$ for each chirality of $S O(6)$

$$
J_{+}^{a} \equiv \frac{1}{2} \eta_{A B}^{a} E^{A} \wedge E^{B}, \quad J_{-}^{a} \equiv \frac{1}{2} \bar{\eta}_{A B}^{a} E^{A} \wedge E^{B} .
$$

It is easy to derive the following useful identity

$$
J_{ \pm}^{a} \wedge J_{ \pm}^{b} \wedge J_{ \pm}^{c}= \pm \frac{1}{2} d^{a b c} \operatorname{vol}(g)
$$

where $\operatorname{vol}(g)=\sqrt{g} d^{6} x$. Also introduce a nondegenerate 2-form on $M$

$$
\Omega=\frac{1}{2} \bar{I}_{A B} E^{A} \wedge E^{B}=E^{1} \wedge E^{2}+E^{3} \wedge E^{4}+E^{5} \wedge E^{6}
$$

where $\bar{I} \equiv i \sigma^{2} \otimes \mathbf{I}_{3}$. This two-form can be wedged with the Hodge star to construct a diagonalizable operator on $\Lambda^{2} T^{*} M$ as follows

$$
*_{\Omega} \equiv *(\bullet \wedge \Omega): \Lambda^{2} T^{*} M \stackrel{\bullet \wedge \Omega}{\longrightarrow} \Lambda^{4} T^{*} M \stackrel{*}{\longrightarrow} \Lambda^{2} T^{*} M
$$

by $*_{\Omega}(\alpha)=*(\alpha \wedge \Omega)$ for $\alpha \in \Lambda^{2} T^{*} M$. The $15 \times 15$ matrix representing $*_{\Omega}$ turns out to have eigenvalues 2,1 and -1 with eigenspaces of dimension 1,6 and 8 , respectively. On any sixdimensional Riemannian manifold $M$, the space of 2-forms $\Lambda^{2} T^{*} M$ can thus be decomposed into three subspaces [Q]

$$
\Lambda^{2} T^{*} M=\Lambda_{1}^{2} \oplus \Lambda_{6}^{2} \oplus \Lambda_{8}^{2}
$$


where $\Lambda_{1}^{2}$ and $\Lambda_{6}^{2}$ are locally spanned by

$$
\begin{aligned}
& \Lambda_{1}^{2}=2\left(J_{+}^{3}+\frac{1}{\sqrt{3}} J_{+}^{8}+\frac{1}{\sqrt{6}} J_{+}^{15}\right)=\Omega \\
& \Lambda_{6}^{2}=\left\{J_{+}^{1}, J_{+}^{2}, J_{+}^{4}, J_{+}^{5}, J_{+}^{9}, J_{+}^{10}\right\}
\end{aligned}
$$

and $\Lambda_{8}^{2}$ by

$$
\Lambda_{8}^{2}=\left\{J_{+}^{6}, J_{+}^{7}, J_{+}^{11}, J_{+}^{12}, J_{+}^{13}, J_{+}^{14}, K_{+}, L_{+}\right\}
$$

with $K_{+} \equiv \frac{1}{\sqrt{3}}\left(J_{+}^{8}-\sqrt{2} J_{+}^{15}\right)$ and $L_{+} \equiv \frac{2}{\sqrt{3}}\left(-\frac{1}{2} J_{+}^{3}+\frac{1}{\sqrt{3}} J_{+}^{8}+\frac{1}{\sqrt{6}} J_{+}^{15}\right)$. A similar decomposition can be done with the negative chirality basis $J_{-}^{a}$.

In conclusion, $J_{+}^{a}$ and $J_{-}^{a}$ in (5.17) serve as independent basis of two-forms in the space of positive chirality and negative chirality, respectively. Note that $F^{(+) a}=\frac{1}{2} F_{A B}^{(+) a} E^{A} \wedge E^{B}$ in (5.2]) and $F^{(-) a}=\frac{1}{2} F_{A B}^{(-) a} E^{A} \wedge E^{B}$ in (5.13) are field strengths of $S U$ (4) gauge fields defined in the space of positive chirality and negative chirality, respectively. Therefore one can expand the $S U(4)$ field strengths in the classes $\mathbb{A}$ and $\mathbb{B}$ in terms of the two-form basis in ([.]ل)

$$
\begin{aligned}
& \mathbb{A}: \quad F_{A B}^{(+) a}=f_{(++)}^{a b} \eta_{A B}^{b}, \\
& \mathbb{B}: \quad F_{A B}^{(-) a}=f_{(--)}^{a b} \bar{\eta}_{A B}^{b} .
\end{aligned}
$$

Consequently, we expand the six-dimensional Riemann curvature tensors according to their sixdimensional chirality class for two different classes [Q]:

$$
\begin{array}{ll}
\mathbb{A}: & R_{A B C D}=f_{(++)}^{a b} \eta_{A B}^{a} \eta_{C D}^{b}, \\
\mathbb{B}: & R_{A B C D}=f_{(--)}^{a b} \bar{\eta}_{A B}^{a} \bar{\eta}_{C D}^{b} .
\end{array}
$$

In order to define $\Omega$-self-duality equations in Eq. (5.20), it is useful to introduce the following operator acting on $6 \times 6$ antisymmetric matrices defined by [Q]

$$
P_{ \pm}^{A B C D} \equiv \frac{1}{4}\left(\delta_{A C} \delta_{B D}-\delta_{A D} \delta_{B C}\right) \pm \frac{1}{8} \varepsilon^{A B C D E F} \bar{I}_{E F}=P_{ \pm}^{C D A B}
$$

One can deduce by a straightforward calculation the following properties

$$
P_{ \pm}^{A B E F} P_{ \pm}^{E F C D}=P_{ \pm}^{A B C D}+\frac{1}{8} \bar{I}_{A B} \bar{I}_{C D}, \quad P_{ \pm}^{A B E F} P_{\mp}^{E F C D}=-\frac{1}{8} \bar{I}_{A B} \bar{I}_{C D}
$$

The action of the operator (5.29) on the two-form $F$ in (5.J6) is given by

$$
P_{ \pm}^{A B C D} F_{C D}=\frac{1}{2}\left(F_{A B} \pm \frac{1}{4} \varepsilon^{A B C D E F} F_{C D} \bar{I}_{E F}\right)
$$

or in terms of form notation

$$
2 P_{ \pm} F=F \pm *(F \wedge \Omega)=F \pm *_{\Omega} F .
$$

Hence we see that, if $F \in \Lambda_{8}^{2}=\left\{F \mid P_{+} F=0\right\}$, it satisfies the $\Omega$-anti-self-duality equation [प]]

$$
F=-*(F \wedge \Omega)
$$


whereas $F \in \Lambda_{6}^{2}=\left\{F \mid P_{-} F=0\right\}$ satisfies the $\Omega$-self-duality equation

$$
F=*(F \wedge \Omega) .
$$

Now we are ready to identify the gauge theory object corresponding to Calabi-Yau manifolds in six dimensions [Q] Suppose that $(M, \Omega, g)$ is a Kähler manifold. One can show that the Kählar condition, $d \Omega=0$, is equivalent to the result that the gauge fields in (5.55) take values in $U(3)$ Lie algebra. This immediately leads to the conclusion that

$$
\begin{aligned}
F^{(+) a} & =d A^{(+) a}-\frac{1}{2} f^{a b c} A^{(+) b} \wedge A^{(+) c} \\
& =f_{(++)}^{a b} J_{+}^{b} \in \Lambda_{8}^{2} \oplus \Lambda_{1}^{2}
\end{aligned}
$$

where the field strengths $F^{(+) a}, a=0,1, \cdots, 8$ are defined by Eq. ([D. $(2)$ with $U(3)$ generators $T^{a}$. As is well-known [0, [0]], the Ricci-tensor of a Kähler manifold is the field strength of the $U(1)$ part of the spin connection which is given by $F^{(+) 0} \in \Lambda_{1}^{2}$ and $F^{(+) \hat{a}} \in \Lambda_{8}^{2}, \hat{a}=1, \cdots, 8$ belong to the $S U$ (3) part. In particular, as $F^{(+) \hat{a}} \in \Lambda_{8}^{2}$, they satisfy the $\Omega$-anti-self-duality equation (5.33) known as the Hermitian Yang-Mills equations (or Donaldson-Uhlenbeck-Yau equations) [ए, [1] ]

$$
F^{(+) \hat{a}}=-*\left(F^{(+) \hat{a}} \wedge \Omega\right), \quad \hat{a}=1, \cdots, 8 .
$$

The same formulae can be obtained for the type $\mathbb{B}$ case and Calabi-Yau manifolds for the type $\mathbb{B}$ case are also described by the Hermitian Yang-Mills equations

$$
F^{(-) \hat{a}}=-*\left(F^{(-) \hat{a}} \wedge \Omega\right), \quad \hat{a}=1, \cdots, 8 .
$$

In summary, the Kähler condition projects the 't Hooft symbols to $U(3)$-valued ones and results in the reduction of the gauge group from $S U(4)$ to $U(3)$. After imposing the Ricci-flat condition or the trivial first Chern class, $F^{(+) 0}=d A^{(+) 0}=0$, the gauge group is further reduced to $S U(3)$. Remaining spin connections are $S U(3)$ gauge fields and satisfy the Hermitian Yang-Mills equation (536) or (5.37). As a Kähler manifold with trivial first Chern class is a Calabi-Yau manifold, this means that the Calabi-Yau manifold is described by the Hermitian Yang-Mills equation (5.33) whose solution is known as Hermitian Yang-Mills instantons [ए0]. Consequently, we find that six-dimensional Calabi-Yau manifolds are equivalent to Hermitian Yang-Mills instantons in $S U$ (3) Yang-Mills gauge theory [Q].

The equivalence between Calabi-Yau manifolds and $S U(3)$ Hermitian Yang-Mills instantons can be more clarified by considering $S U$ (4) Yang-Mills theory defined on a Riemannnian manifold $(M, g)$ whose action is given by

$$
S_{Y M}=-\frac{1}{2 g_{Y M}^{2}} \int_{M} d^{6} x \sqrt{g} g^{M P} g^{N Q} \operatorname{Tr} F_{M N} F_{P Q}
$$

One can rewrite the action $(5.38)$ as

$$
\begin{aligned}
S_{Y M}=-\frac{1}{4 g_{Y M}^{2}} \int_{M} d^{6} x \sqrt{g} \operatorname{Tr}[ & \left(F_{A_{1} B_{1}} \pm \frac{1}{4} \varepsilon^{A_{1} B_{1} A_{2} B_{2} A_{3} B_{3}} F_{A_{2} B_{2}} \bar{I}_{A_{3} B_{3}}\right)^{2}-\frac{1}{2}\left(\bar{I}_{A B} F^{A B}\right)^{2} \\
& \left.\mp \frac{1}{2} \varepsilon^{A B C D E F} F_{A B} F_{C D} \bar{I}_{E F}\right]
\end{aligned}
$$


using the following formula

$$
\begin{aligned}
\left(P_{ \pm} F\right)^{2} & =\left(P_{ \pm}^{A_{1} B_{1} A_{2} B_{2}} F_{A_{2} B_{2}}\right)\left(P_{ \pm}^{A_{1} B_{1} A_{3} B_{3}} F_{A_{3} B_{3}}\right) \\
& =\frac{1}{4}\left(F_{A_{1} B_{1}} \pm \frac{1}{4} \varepsilon^{A_{1} B_{1} A_{2} B_{2} A_{3} B_{3}} F_{A_{2} B_{2}} \bar{I}_{A_{3} B_{3}}\right)^{2} \\
& =P_{ \pm}^{A B C D} F_{A B} F_{C D}+\frac{1}{8}\left(\bar{I}_{A B} F^{A B}\right)^{2} \\
& =\frac{1}{2} F_{A B} F^{A B} \pm \frac{1}{8} \varepsilon^{A B C D E F} F_{A B} F_{C D} \bar{I}_{E F}+\frac{1}{8}\left(\bar{I}_{A B} F^{A B}\right)^{2},
\end{aligned}
$$

which can be derived by using the projection operator (5.29) and the identity (5.30). The above action may be written in a more compact form [Q] as

$$
\begin{aligned}
S_{Y M}= & -\frac{1}{4 g_{Y M}^{2}} \int_{M} d^{6} x \sqrt{g} \operatorname{Tr}\left[\left(F_{A B} \pm *(F \wedge \Omega)_{A B}\right)^{2}-\frac{1}{2}\left(\bar{I}_{A B} F^{A B}\right)^{2}\right] \\
& \pm \frac{1}{g_{Y M}^{2}} \int_{M} d^{6} x \operatorname{Tr} F \wedge F \wedge \Omega
\end{aligned}
$$

where $\Omega$ is the two-form of rank 6 defined in Eq. (5.

Using the fact

$$
\operatorname{Tr} F \wedge F=d \operatorname{Tr}\left(A \wedge F-\frac{1}{3} A \wedge A \wedge A\right) \equiv d K,
$$

one can see that the last term in Eq. (5.41) is a topological term, i.e.,

$$
\operatorname{Tr} F \wedge F \wedge \Omega=d(K \wedge \Omega)
$$

if and only if the two-form $\Omega$ is closed, i.e. $d \Omega=0$. In other words, when $M$ is a Kähler manifold, the last term in Eq. (5.4II) depends only on the topological class of the Kähler-form $\Omega$ and the vector bundle $E$ on $M$. In order to keep the positive definiteness of the Yang-Mills action (5.4J) after separating the topological term, it is necessary to impose the following requirement

$$
\bar{I}_{A B} F^{A B}=0 .
$$

After the condition (5.44), the action (5.41) is now positive definite up to a topological term and the minimum action can be achieved in a configuration satisfying the equations

$$
F_{A B} \pm *(F \wedge \Omega)_{A B}=0 .
$$

The above $\Omega$-self-duality equations together with Eq. (5.44) implies the so-called DonaldsonUhlenbeck-Yau equation

$$
F \wedge \Omega^{2}=0 .
$$

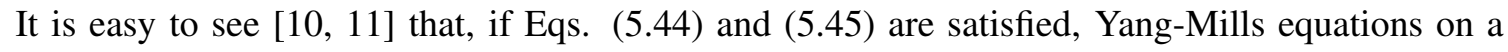
Kähler manifold given by

$$
g^{M N} D_{M} F_{N P}=0
$$

are automatically satisfied.

Finally it can be shown [Q] that Hermitian Yang-Mills instantons obeying (5.44) and (5.45) are connections of a stable holomorphic vector bundle in $S U(3)$ Yang-Mills gauge theory with the trivial first Chern class. This is precisely the result for Calabu-Yau manifolds [ए0]]. 
Acknowledgments The author would like to thank the organizers of the Corfu Summer Institute 2011 School and Workshops on Elementary Particle Physics and Gravity and in particular, G. Zoupanos, for a stimulating meeting and an enjoyable time at Corfu. We also thank Jungjai Lee, Siyoung Nam, John J. Oh, Chanyong Park and Sangheon Yun for collaborations and helpful discussions.

\section{References}

[1] C.W. Misner, K.S. Thorne and J.A. Wheeler, Gravitation, W.H. Freeman and Company, New York, 1973.

[2] J.J. Oh, C. Park and H.S. Yang, JHEP 04 (2011) 087 [arXiv: 1101.1357 ].

[3] J.J. Oh and H.S. Yang, Einstein Manifolds As Yang-Mills Instantons, [arXiv: 1101.5185$].$

[4] J. Lee, J.J. Oh and H.S. Yang, JHEP 12 (2011) 025 [arXiv: 1109.6644 ].

[5] T. Eguchi, P.B. Gilkey and A.J. Hanson, Phys. Rep. 66 (1980) 213.

[6] H.B. Lawson, Jr. and M.-L. Michelsohn, Spin Geometry, Princeton Univ. Press, New Jersey, 1989.

[7] A.L. Besse, Einstein Manifolds, Springer-Verlag, Berlin, 1987.

[8] S.K. Donaldson and P.B. Kronheimer, The Geometry of Four-Manifolds, Oxford Univ. Press, Oxford, 1990.

[9] H.S. Yang and S. Yun, Calabi-Yau Manifolds, Hermitian Yang-Mills Instantons and Mirror Symmetry, [arXiv:1107.2095].

[10] M.B. Green, J.H. Schwarz and E. Witten, Superstring Theory, Vol. 2, Cambridge Univ. Press, New York, 1987.

[11] G. Tian, Annals Math. 151 (2000) 193 [arXiv:math/0010015].

[12] P. Candelas, G.T. Horowitz, A. Strominger and E. Witten, Nucl. Phys. B258 (1985) 46. 